\title{
Magnetic Properties of Iron Nanoparticles Distributed in Polymer Matrix: Theoretical and Experimental Approach
}

\author{
M.A. Ramazanov ${ }^{a, *}$, H.A. Shirinova ${ }^{a}$, F.V. Hajiyeva $^{a}$, \\ J.R. Sultanova ${ }^{b}$ And R.A. Alizade ${ }^{c}$ \\ ${ }^{a}$ Baku State University, Physics Faculty, Z. Khalilov Str. 23, Baku, AZ1148, Azerbaijan \\ ${ }^{b}$ Azerbaijan Aerocosmic Agency, Azerbaijan \\ ${ }^{c}$ Institute of Physics of Azerbaijan National Academy of Sciences, \\ pr. H. Javid Ave. 33, Baku, AZ1143, Azerbaijan
}

Received: 07.04.2020 \& Accepted: 25.08.2020

Doi: $10.12693 /$ APhysPolA.138.787

*e-mail: mamed_r50@mail.ru

\begin{abstract}
A PP + Fe nanocomposite's phase identification, morphology, nanoscale imaging and magnetic structure have been determined with the use of the X-ray diffraction, scanning electron microscopy, atomic force microscopy and magnetic force microscopy techniques, respectively. In a polymer matrix, iron nanoparticles are found to be randomly distributed and their distribution was described by the log-normal function. The theoretically determined dependence of the particles' size on concentration showed good agreement with the scanning electron microscopy results. The present study showed that the magnetic and geometric sizes of Fe nanoparticles in the polymer matrix differ from each other. Additionally, the magnetic size of the $\mathrm{Fe}$ nanoparticles of the identical geometric size decreases with increasing filler concentration in the polymer matrix. The increase of Fe nanoparticles' concentration presumably leads to oxidation of the surface layer which is likely non-magnetic. The magnetic behaviour of the $\mathrm{PP}+\mathrm{Fe}$ nanocomposite depending on iron content was investigated both theoretically and experimentally. The results of the magnetic measurement indicated the presence of a natural oxide layer in addition to pure iron. The comparison of the theoretical curve of $M(H)$ with that of the magnetic measurement clearly showed that the obtained curves share the same qualitative feature. In both cases, for all concentrations of the nanoparticles, an open hysteresis loop characteristic for multi-domain particles was observed. The qualitative difference between the experiment and theoretical calculations was explained by the influence of several factors including the microstructure of the medium and the magnetic domain structure of the iron nanoparticles in the polymer matrix.
\end{abstract}

topics: polymer nanocomposite, size distribution, magnetization

\section{Introduction}

It has long been known that magnetic properties of nanomaterials, similarly to all other functional properties, strongly depend on the system size $[1,2]$. Magnetic nanoparticles also possess a characteristic domain structure which, in turn, is directly related to the nanoparticles' size. The correlation between the particle size and the domain structure of some magnetic nanoparticles was investigated in $[3,4]$. However, when their size approaches the size of a single domain, the magnetic nanoparticles exhibit new unusual properties.

As a matter of fact, the domain size and the size distribution of nanoparticles depends on several factors, i.e., the amount of solvent, the amount of precursor, the density of the compounds, the viscosity of the system, etc. Moreover, if nanoparticles are distributed in a matrix, their size dramatically depends on their concentration in the medium $[5,6]$. Numerous researches show that unique properties appear only in the case of a low content of dispersed phase [7-11]. There is, however, no information on how to describe the size distribution of nanoparticles in the matrix and with what kind of regularity the size changes when the nanoparticles' volume content increases. Assuming the magnetic properties are strongly dependent on the particle size, the task of finding the relationship between the geometric sizes, the concentration and the domain structures of nanoparticles in the polymer matrix is of considerable importance.

In this work, we used the combination of several modern techniques, such as the X-ray diffraction (XRD), scanning electron microscopy (SEM), atomic force microscopy (AFM), magnetic force microscopy (MFM) and magnetometer measurements. Furthermore, the theoretical background regarding 
the magnetic properties of iron nanoparticles dispersed in the polymer matrix was investigated. The results obtained by the measurement and the outcome of the theoretical calculation were comparatively analyzed. Studies of this subject, although rare, are available in the literature, e.g. [12]. However, the correlation between concentration, size and magnetic response of metal nanoparticles distributed in a polymer matrix has not been deeply investigated so far.

Our research was designed to predict the growing size of metal nanoparticles in a host matrix and the magnetic properties of a nanocomposite depending on the nanoparticles concentration with the help of theoretical calculation, before an experimental measurement.

\section{Experimental}

\subsection{Materials}

The isotactic polypropylene (PP brand Sigma Aldrich $\mathrm{P}$ code 1001326963) is characterized with the density of $0.9 \mathrm{~g} / \mathrm{ml}$ at $25^{\circ} \mathrm{C}$, the refractive index of $n_{20} / D=1.49$, the transition temperature $T_{g}=-26{ }^{\circ} \mathrm{C}$, the average $M_{w} \approx 250000 \mathrm{~mol} \mathrm{ob}-$ tained by gel permeation chromatography (GPC), the auto ignition temperature is $356.7^{\circ} \mathrm{C}$, and the $M_{p}=189^{\circ} \mathrm{C}$. Iron nanoparticles were prepared using the electro-explosive technology.

\subsection{Synthesis of polymer nanocomposites}

$\mathrm{PP}+\mathrm{Fe}$ based nanocomposite samples were prepared by the ex situ method [13]. PP polymer was solved in the toluene solvent at $120^{\circ} \mathrm{C}$. Then, the Fe nanoclusters with the $20-100 \mathrm{~nm}$ sizes were added into the polymer solution and stirred within $2 \mathrm{~h}$ at $30-40^{\circ} \mathrm{C}$ until obtaining a homogeneous mixture. In order to remove the solvent, samples were dried in a vacuum oven during $24 \mathrm{~h}$. Thin film nanocomposite samples with various $\mathrm{Fe}$ nanoparticles volume content were obtained by hot pressing at $165^{\circ} \mathrm{C}$ under $15 \mathrm{MPa}$ pressure within 4 min on further cooling to room temperature $[13,14]$.

\subsection{Methods of research}

\subsection{1. $X R D$}

The X-ray diffraction analysis was performed on Rigaku Mini Flex 600 XRD diffractometer at ambient temperature. In all the cases, $\mathrm{Cu} \mathrm{K}_{\alpha}$ radiation from a $\mathrm{Cu}$ X-ray tube (run at $15 \mathrm{~mA}$ and $30 \mathrm{kV}$ ) was used. The samples were scanned in the Bragg angle $2 \mathrm{~h}$ range of $20-70^{\circ} \mathrm{C}$.

\subsubsection{SEM analysis}

The scanning electron microscopy and energydispersive spectrum analysis of nanocomposite samples were taken on scanning electron microscope JEOL JSM-7600F at an accelerating voltage of $15.0 \mathrm{kV}$, the SEI regime. The resolution of SEM JSM-7600F at an accelerating voltage of $15.0 \mathrm{kV}$ was $1.0 \mathrm{~nm}$.

\subsubsection{AFM analysis}

The morphology of the nanocomposites was studied using atomic force microscopy Integra Prima (NT-MDT, Zelenograd). Special silicon cantilevers, fabricated by the plasma etching method with the needle radius of curvature of $20 \mathrm{~nm}$ and the resonance frequency of $1-5 \mathrm{~Hz}$, were used for the scan. The scan size was $2 \times 2 \mathrm{~mm}^{2}$. The measurements were performed in the semicontact microscopy mode in the air. A needle change of the cantilever oscillation amplitude was fixed, determining the surface topography. The scanning speed and the number of scanned lines of the image were, respectively, 256 and $1969 \mathrm{~Hz}$.

The Nova Power Script software was used for AFM/MFM image processing. The linear dimension estimation error in AFM is $\pm 0.5 \%$.

\subsubsection{Magnetic force microscopy}

Magnetic force microscopy (MFM) images were obtained with Prima AFM Integra Prima using a two-pass technique, at the so-called "lifting" mode. The magnetic force microscopy shows the spatial pattern of the magnetic force on the sample surface with the assistance of the MFM probe coated with a thin ferromagnetic film. A MFM image is obtained via a two-pass method. On the first pass, the relief of the sample is determined using a contact or intermittent-contact regime. On the second pass, the probe is lifted and only long-range magnetic forces are able to affect the probe. At the same time, the MFM image is obtained by measuring the amplitude shift or the cantilever oscillation phase which occurs under the influence of the magnetic force between the sample surface and a magnetized MFM cantilever. Images of magnetic force microscopy contain information about the location of the magnetic domains on the sample surface. The linear dimension estimation error in MFM was $\pm 1.2 \%$.

\subsection{5. $M(H)$ measurements}

Magnetization curves were acquired at $300 \mathrm{~K}$ (room temperature) by a Quantum Design SQUID magnetometer in the field range of $\pm 50 \mathrm{kOe}$. The magnetization is reported per gram of the measured sample. The sensitivity of the Quantum Design SQUID magnetometer is $5 \times 10^{-8}$ emu. The field amplitude equals $0.005-15$ Oe.

\subsubsection{Calculation techniques}

The calculations were carried out using programs written in the algorithmic Fortran language and implemented by Visual Fortran-6.6. Graphs were prepared using a data processing software, Origin-8. 


\section{Results and discussion}

Figure 1 presents the diffraction pattern of pure iron (Fe) nanoparticles. As the graph shows, the peaks at $2 \theta$ values of $44.72^{\circ}, 65.10^{\circ}, 82.42^{\circ}$ and $116.43^{\circ}$ correspond to [110], [220], [211] and [310] planes of iron (card number-ICDD no. 00-006-0696), respectively. Figure 2 demonstrates the XRD patterns of $\mathrm{PP}+\mathrm{Fe}$ nanocomposites. It is clear that with increasing the volume content of Fe nanoclusters in the PP matrix, the degree of crystallinity increases.

Figure 3 represents the SEM images of $\mathrm{PP}+\mathrm{Fe}$ nanocomposites. The number of agglomerates rises with increasing the volume content of nanoparticles in the polymer matrix. As demonstrated in Fig. 3, for 0.1, 1 and 5\% volume contents of the filler, the average sizes of magnetite nanoclusters in the matrix are 47,54 and $77 \mathrm{~nm}$, respectively.

Iron nanoparticles are randomly distributed in the polymer matrix. Table I demonstrates some distribution parameters of $\mathrm{Fe}$ nanoparticles in the PP matrix depending on their concentration. These parameters include the minimum and maximum size of iron nanoparticles in the matrix $\left(d_{\min }\right.$ and $\left.d_{\max }\right)$, their mean size $(\bar{d})$, standard deviation $(\sigma)$, asymmetry parameter $(a)$, excess parameter $(\varepsilon)$ and polydispersity index (PDI).

A normal distribution function is not suitable for describing the distribution of Fe nanoparticles in the PP matrix since in normal distribution, the asymmetry parameter and excess are zero (see Table I). Otherwise, there would be a deviation

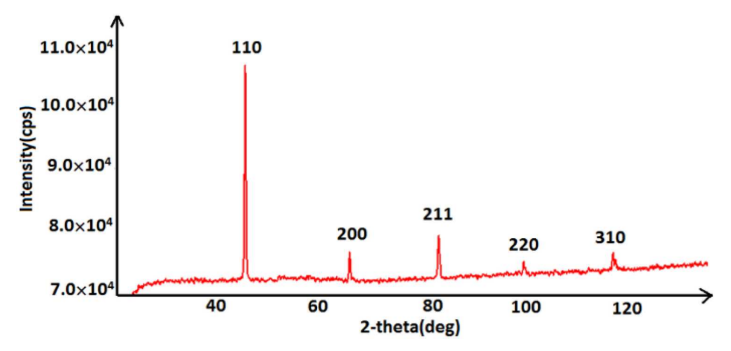

Fig. 1. XRD pattern of pure iron nanoparticles.

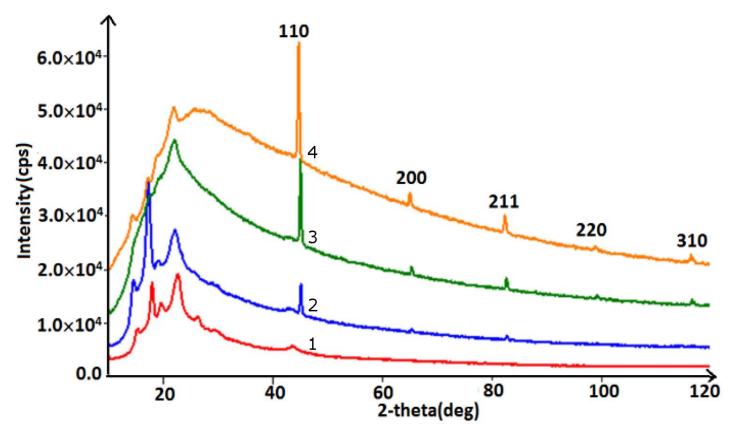

Fig. 2. XRD patterns of pure $\mathrm{PP}(1)$ and $\mathrm{PP}+\mathrm{Fe}$ nanocomposites: $\mathrm{PP}+0.1 \% \mathrm{Fe}(2), \mathrm{PP}+5 \% \mathrm{Fe}(3)$, $\mathrm{PP}+10 \% \mathrm{Fe}(4)$.
TABLE I

Distribution parameters of $\mathrm{Fe}$ nanoparticles in $\mathrm{PP}$ matrix according to SEM investigation.

\begin{tabular}{c|c|c|c|c|c|c}
\hline \hline $\begin{array}{c}\text { Filler } \\
\text { concentration } \\
\text { in PP matrix }\end{array}$ & $\begin{array}{c}\left(d_{\min }, d_{\max }\right) \\
{[\mathrm{nm}]}\end{array}$ & $\bar{d}[\mathrm{~nm}]$ & $\sigma$ & $a$ & $\varepsilon$ & PDI \\
\hline $0.1 \% \mathrm{Fe}$ & $(14.2,73.7)$ & 46.48 & 1.24 & 0.21 & -0.45 & 1.24 \\
$10 \% \mathrm{Fe}$ & $(6.2,132.0)$ & 77.91 & 2.23 & 0.19 & 0.49 & 1.29
\end{tabular}
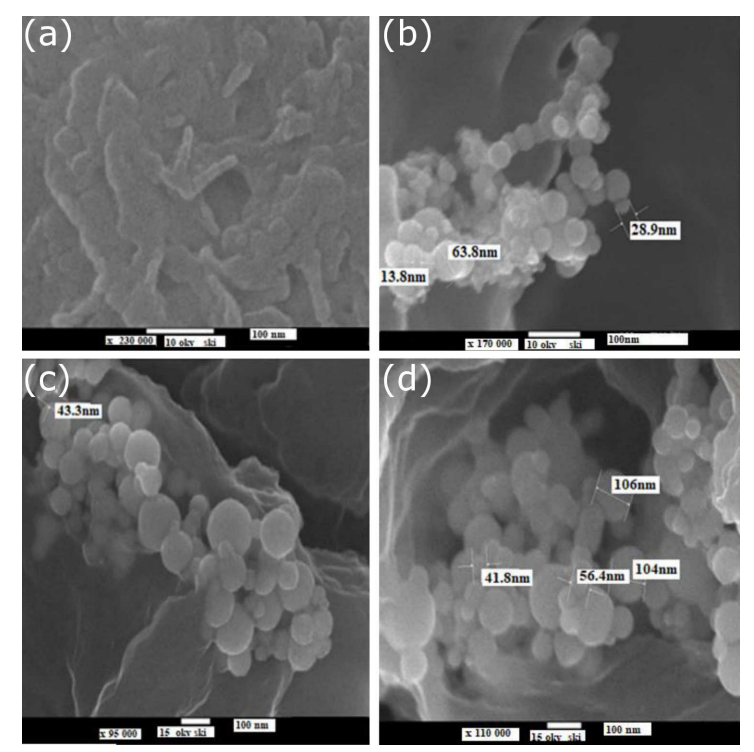

Fig. 3. SEM image of $\mathrm{PP}+\mathrm{Fe}$ based nanocomposites: (a) $\mathrm{PP}$, (b) $\mathrm{PP}+0.1 \% \mathrm{Fe}$, (c) $\mathrm{PP}+1 \% \mathrm{Fe}$, (d) $\mathrm{PP}+5 \% \mathrm{Fe}$.

from the normal distribution [15]. For this reason, the Galton distribution function was used for the description of Fe distribution in the PP matrix [16]. The probability density function, when $x>0$, is defined as

$$
N(\ln (x), \mu, \sigma)=\frac{1}{\sqrt{2 \pi \sigma^{2}}} \exp \left(-\frac{(\ln (x)-\mu)^{2}}{2 \sigma^{2}}\right)
$$

with the mean $\mu$ and the standard deviation $\sigma$. Figure 4 represents Fe nanoparticles size distribution function in the polymer matrix. The size distribution function was obtained by the analysis of the SEM images. The size of the majority of nanoparticles in $\mathrm{PP}+0.1 \% \mathrm{Fe}$ nanocomposites is $\approx 45 \mathrm{~nm}$, while the average size of the iron nanoparticles in $\mathrm{PP}+10 \% \mathrm{Fe}$ nanocomposite is $\approx 75 \mathrm{~nm}$.

The dependence of the Fe nanoparticles' average diameter on their concentration in the polymer matrix was also investigated (see Fig. 5). The theory of coagulation of nanoparticles in solutions at their low concentrations is known. According to this theory, the number of particles during coagulation decreases according to the law [17].

In Fig. 5a, the theoretically determined particle size (diameter) dependence on the low volume fraction of $\mathrm{Fe}$ has a linear character. However, 


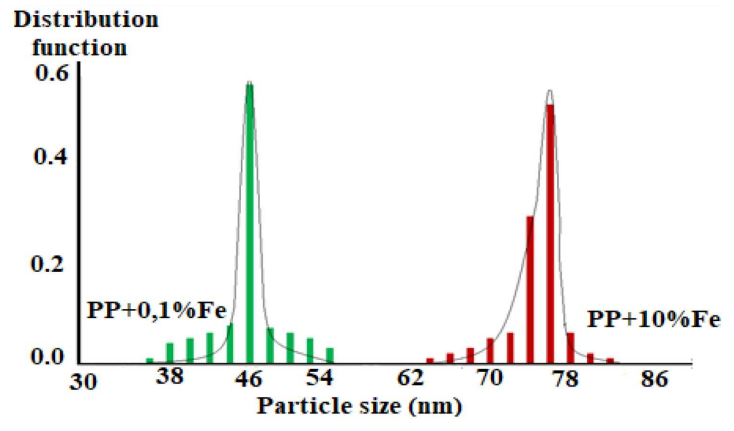

Fig. 4. The log-normal size distribution function of Fe nanoclusters in the PP polymer matrix.

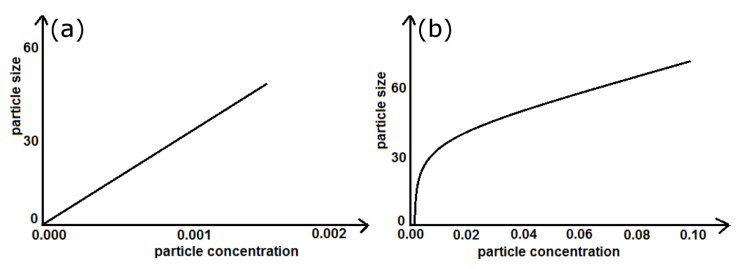

Fig. 5. Dependence of the diameter of Fe nanoclusters on their concentration in polymer PP matrix: (a) low content of the filler, (b) high content of the filler.

for a higher concentration of the Fe nanoparticles in the PP matrix, this dependence is complicated. Note that at high concentrations, saturation is achieved reaching the maximum coagulated particle diameter which can be described with the square root function. The theoretically determined dependence of $d\left(\phi_{v}\right)$ demonstrates good agreement with the obtained SEM results. According to the SEM analysis, the average sizes of the Fe nanoparticles in the PP matrix are 47, 54, 77 and $78 \mathrm{~nm}$ for $\mathrm{PP}+0.1 \% \mathrm{Fe}, \mathrm{PP}+1 \% \mathrm{Fe}, \mathrm{PP}+5 \% \mathrm{Fe}, \mathrm{PP}+10 \% \mathrm{Fe}$ nanocomposites, respectively. Based on the obtained results, it can be concluded that with increasing the filler content in the polymer matrix, the size of particles reaches the saturation value [18].

With the help of the AFM and MFM methods, the geometric and magnetic sizes of the Fe nanoparticles in the polymer matrix at the atomic level were investigated. Figure 6 demonstrates 2D AFM images and histograms of nanocomposites with different filler content. As it can be observed, the root mean square roughness of samples varies between 30 and $40 \mathrm{~nm}$. According to AFM images, it is clear that the size of the nanoparticles in the matrix increases with rising concentration. Hence, this result proves the SEM analysis results.

Based on MFM investigation, it was shown that iron nanoparticles became magnetized when the magnetic probe tip approached them. Magneticforce-microscopic studies of nanocomposites confirm that iron nanoparticles, distributed in the matrix, form domains in the local regions and form a local magnetic field around themselves

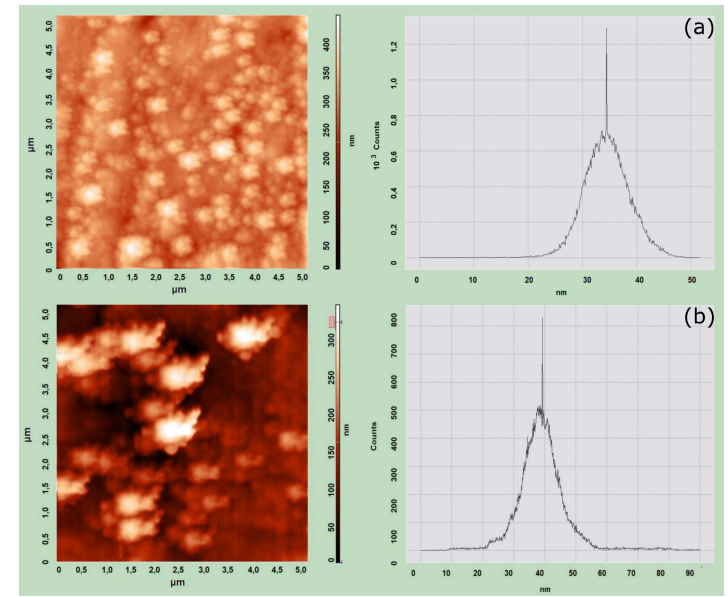

Fig. 6. 2D AFM images and histogram of $\mathrm{PP}+\mathrm{Fe}$ nanocomposites: (1) $\mathrm{PP}+0.1 \% \mathrm{Fe},(2) \mathrm{PP}+5 \% \mathrm{Fe}$.

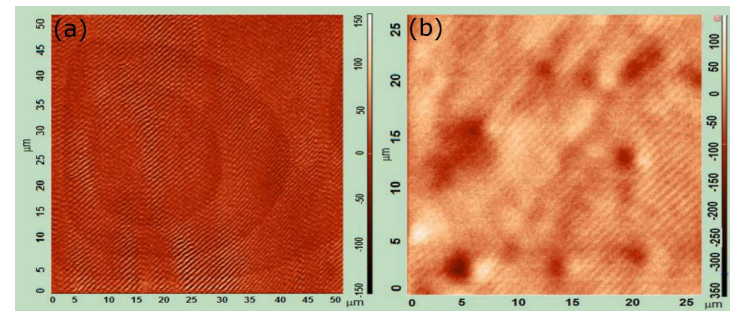

Fig. 7. Domain structure of $\mathrm{PP}+\mathrm{Fe}$ based magnetic nanocomposites.

(see Fig. 7). The size of these domains varies in the range of 150-400 $\mathrm{nm}$. In fact, this proves that the magnetic and geometric sizes of nanoclusters indeed differ from each other [19]. The relation between the geometric size $\left(d_{g}\right)$ and magnetic size $\left(d_{m}\right)$ of Fe nanoparticles in the polymer matrix has been theoretically investigated.

The relation between the $\phi_{g}$ geometric (volume) and the $\phi_{m}$ magnetic concentration of a particle is given accordingly

$$
\begin{aligned}
& \frac{\phi_{g}}{\phi_{m}}=\frac{d_{g}^{3}}{\left(d_{g}+x\right)^{3}}=\alpha, \\
& x^{3}-3 d_{g} x^{2}+3 d_{g}^{2} x-(1-\alpha) d_{g}^{3}=0 .
\end{aligned}
$$

Here, $d_{m}=d_{g}+x$. In order to solve (3), we used the Cardan method [20]. The obtained results from the cubic equation for iron nanoparticles are given in Table II. The calculated magnetic size of the Fe nanoparticles in the polymer matrix depends not only on the geometric size of the nanoparticles but also on their concentration (volume fraction) in the matrix. However, in all cases, the magnetic size of the particles is always bigger than their geometric size. This result indicates a good agreement with AFM investigations.

Table II also demonstrates that the magnetic size of the Fe nanoparticles having the identical geometric size (for example $30 \mathrm{~nm}$ ) decreases with increasing filler concentration in the PP matrix. The rise 
TABLE II

Relation between magnetic and geometric sizes.

\begin{tabular}{c|c|c|c}
\hline \hline $\begin{array}{c}\text { Filler } \\
\text { concentration } \\
\text { in PP matrix } \\
\varphi_{g}[\%]\end{array}$ & $\begin{array}{c}\text { Magnetic } \\
\text { concentration } \\
\varphi_{m}[\%]\end{array}$ & $\begin{array}{c}\text { Geometric } \\
\text { size } \\
d_{g}[\mathrm{~nm}]\end{array}$ & $\begin{array}{c}\text { Magnetic } \\
\text { size } \\
d_{m}[\mathrm{~nm}]\end{array}$ \\
\hline 0.01 & 0.37 & 20 & 66.70 \\
0.01 & 0.32 & 40 & 127.38 \\
0.03 & 1.33 & 30 & 106.90 \\
0.06 & 1.35 & 30 & 85.46 \\
0.07 & 1.35 & 40 & 67.26
\end{tabular}
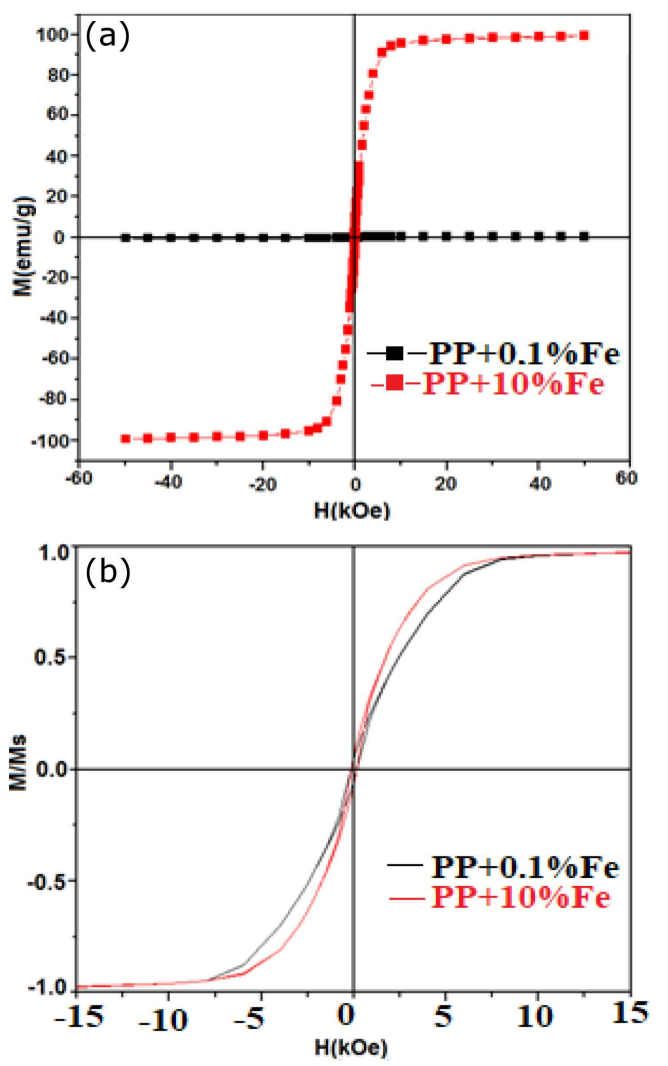

Fig. 8. Magnetization versus magnetic field curves of $\mathrm{PP}+\mathrm{Fe}$ based nanocomposites at $300 \mathrm{~K}$ : (a) magnetization versus magnetic field, (b) normalized magnetization versus magnetic field.

in concentration affects only coagulation. This dramatically changes the magnetic characteristics of the composite. Further, it can be related to oxidizing the surface layer of the Fe nanoparticles and this layer is likely non-magnetic $[21,22]$.

The magnetic properties of $\mathrm{PP}+\mathrm{Fe}$ nanocomposites were studied both experimentally and theoretically. Figure 8 illustrates the experimental hysteresis loops of $\mathrm{PP}+\mathrm{Fe}$ nanocomposite materials with various volume contents of the fillers. The polypropylene matrix itself contributes a weak diamagnetic background. The shape of the magnetization curves suggests the presence of two magnetic phases: a soft and a harder one. The first, soft

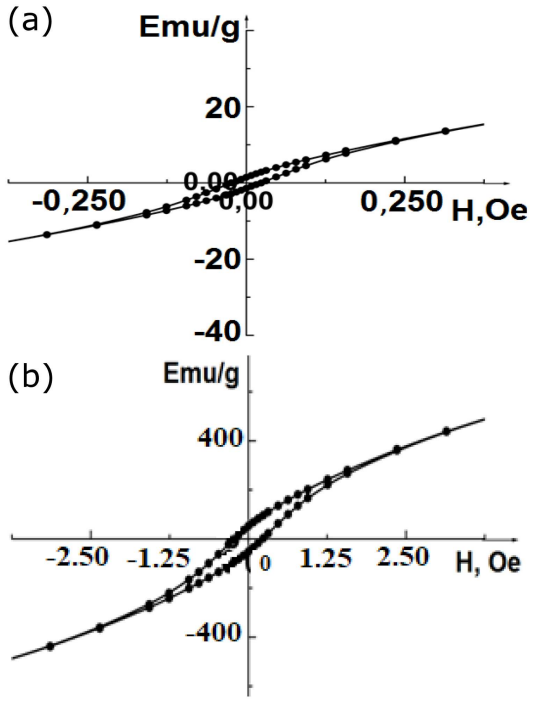

Fig. 9. The theoretically constructed dependence of magnetization on the magnetic field intensity for $\mathrm{PP}+\mathrm{Fe}$ based nanocomposites: (a) $\mathrm{PP}+0.1 \% \mathrm{Fe},(\mathrm{b})$ $\mathrm{PP}+10 \% \mathrm{Fe}$.

magnetic phase is iron nanoparticles. The second, however, is the harder phase which is probably related to a natural oxide layer of iron nanoparticles [23, 24]. This second phase is responsible for the open loop that is observed at low field (Fig. 8b).

According to SQUID magnetometer measurements, the magnetization saturation $M_{s}$ is $0.38 \mathrm{emu} / \mathrm{g}$ and $99.30 \mathrm{emu} / \mathrm{g}$ for $\mathrm{PP}+0.1 \% \mathrm{Fe}$ and $\mathrm{PP}+10 \% \mathrm{Fe}$, respectively. The saturation value of the magnetization $M_{s}$ was obtained through the law of approach to saturation [25, 26]. The magnetization value corresponding to the higher amplitude of the external field $(H)$ was chosen. The value of $M_{s}$ was found by extrapolation to the $M(1 / H)$ graph. Moreover, the value of the coercive field changes from 152 Oe to 143 Oe with increasing filler concentration.

To establish the dependence of $M(H)$ of the $\mathrm{PP}+\mathrm{Fe}$ nanocomposite by using the numerical computing methods $[27,28]$, in the model we took into account the size of nanoparticles and their concentration in the polymer matrix. It stands out from Fig. 9 that the theoretical curve of $M(H)$ possesses the same qualitative feature which was demonstrated by the magnetometer measurement. The main quantitative difference is related to the value of the coercive field. The theoretically calculated values of the coercive field of $\mathrm{PP}+0.1 \% \mathrm{Fe}, \mathrm{PP}+10 \% \mathrm{Fe}$ comprise 0.023 Oe and 0.021 Oe, respectively.

The comparison of Figs. 8 and 9 shows that in both theoretical and experimental dependencies - for all concentrations of the nanoparticles the open hysteresis loop and coercitivity decrease with increasing filler concentration and, respectively, size. This feature is characteristic for multidomain particles (Fig. 10a). 


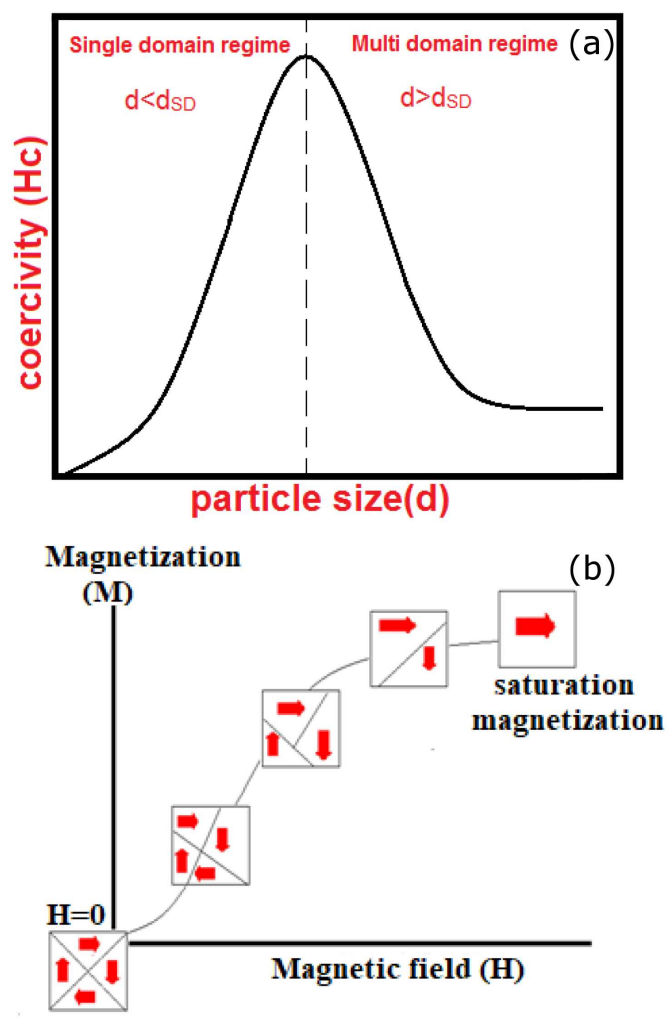

Fig. 10. Coercivity-size dependence of magnetic particles (a), domain arrangements versus external magnetic field (b)

It is known that the value of the coercive field depends on the number of magnetic domains. The domain walls, which are the boundaries between different domains, are characterized with energies, such as the exchange, magnetostatic, anisotropy and magnetoelastic energies [29]. The sum of the energies of the interaction of magnetic domains in a particle, which determines the value of the coercive force, depends on their quantity, temperature, etc.

The size of single-domain nanoparticles of Fe was calculated using [29]:

$$
R_{S D}=\frac{8 \gamma}{\mu_{0} M_{s}^{2}}
$$

where $M_{s}$ is the saturation magnetization and $\gamma$ is the wall energy density that depends on the exchange stiffness and magnetocrystalline anisotropy. For iron nanoparticles of the size of $5.8 \mathrm{~nm}, \gamma$ is about $2.1 \mathrm{erg} / \mathrm{cm}^{3}$. As it can be seen from (4), the domain wall energy depends on the size of the particles. Singe-domain iron particles may consist of up to $35 \times 10^{3}$ atoms. A further increase in the size of particles leads to an increase of the degree of freedom of domain walls and a decrease of coercivity. These facts explain the decrease of the value of the coercive field of the nanocomposites with increasing filler concentration, found both through theoretical and experimental investigation. However, the magnetic structure of the material is highly sensitive to defects of the medium and grain lattice. In the case of polymer nanocomposites, the natural oxide layer, the diamagnetic contribution of the polymer and the presence of the interphase boundary between the filler and the matrix, can play an essential role $[30,31]$. That is why - when the iron nanoparticles are distributed in the polymer matrix - their magnetic orientation depends on several already mentioned factors. Conversely, the ideal case iron nanoparticles in the polymer matrix, even being multi-domain, can easily orientate on the direction of the external magnetic field. As a consequence, the deviation of the measured coercivity and saturation magnetization values from the calculated values occurs.

\section{Conclusion}

Our research was designed to predict the growing size of metal nanoparticles in a host matrix and to determine the magnetic properties of a nanocomposite depending on the nanoparticles concentration with the help of theoretical calculation, before an experimental measurement is conducted. Several modern techniques, such as XRD, SEM, AFM and MFM, were used to study the phase identification, morphology, nanoscale imaging and magnetic structure of the $\mathrm{PP}+\mathrm{Fe}$ nanocomposite, respectively. It was clarified that iron nanoparticles are randomly distributed in the polymer matrix. The log-normal function was used for the description of Fe distribution in the PP matrix. The theoretically determined dependence of the particles size on concentration demonstrated good agreement with the obtained SEM results.

It was observed that the magnetic and geometric sizes of Fe nanoparticles in the polymer matrix differ from each other. Theoretical studies have shown that the magnetic size of the Fe nanoparticles of identical geometric size decreases with increasing filler concentration in the PP matrix. It is presumed that the increase of the Fe nanoparticles concentration leads to oxidation of the surface layer of these particles and it is probably a non-magnetic layer. The magnetic behaviour of the $\mathrm{PP}+\mathrm{Fe}$ nanocomposite depending on iron content was investigated both theoretically and experimentally. The results of the magnetic measurement indicated the presence of a natural oxide layer in addition to pure iron. Moreover, when comparing the theoretical values of $M(H)$ with the magnetic measurement results, it became clear that the curves possess the same qualitative feature. Both in the theoretical and experimental dependencies results, for all concentrations of the nanoparticles, the open hysteresis loop was observed which is characteristic for multidomain particles. The qualitative difference between the experiment and theoretical calculation was explained by the influence of several factors including the microstructure of the medium and the magnetic domain structure of the iron nanoparticles in the polymer matrix. 


\section{References}

[1] Xuemin He, Wei Zhong, Chak-Tong Au, Youwei Du, Nanoscale Res. Lett. 8, 446 (2013).

[2] A.K. Singh, O.N. Srivastava, K. Singh, Nanoscale Res. Lett. 12, 298 (2017).

[3] C. Caizer, "Nanoparticle Size Effect on Some Magnetic Properties", in: Handbook of Nanoparticles, 2015.

[4] Qing Li, Ch.W. Kartikowati, S. Horie, T. Ogi, T. Iwaki, K. Okuyama, Sci. Rep. 7, 9894 (2017).

[5] H.Sh. Dehsar, A.H. Ribeiro, B. Ersöz, W. Tremel, G. Jakob, K. Asadi, CrystEngComm 19, (2017).

[6] F. Silva Bellucci, F.C. Lobato de Almeida, M.A. Lima Nobre, M.A. Rodríguez-Pérez, A. Tabone Paschoalini, A.E. Job, Composites Part B 85, 196 (2016).

[7] M.A. Ramazanov, H.A. Shirinova, F.V. Hajiyeva, Mater. Chem. Phys. 253, 123287 (2020).

[8] L. Di Palma, I. Bavasso, F. Sarasini, Polym. Compos. 39, E1742 (2018).

[9] M.A. Ramazanov, A.M. Maharramov, L. Di Palma, H.A. Shirinova, F.V. Hajiyeva, M.R. Hasanova, J. Ferroelectr. 537, 191 (2018).

[10] A. Tugirumubano, S.H. Go, H.J. Shin, L.K. Kwac, H.G. Kim, Nanomaterials 10, 436 (2020).

[11] A.M. Maharramov, M.A. Ramazanov, R.A. Alizade, P.B. Asilbeyli, Digest J. Nanomater. Biostruct. 8, 1447 (2013).

[12] P. Bender, C. Balceris, F. Ludwig et al., New J. Phys. 19, 073012 (2017).

[13] M.A. Ramazanov, A.M. Maharramov, J.R. Sultanova, F.V. Hajiyeva, U.A. Hasanova, J. Ovon. Res. 12, 193 (2016).

[14] M.A. Ramazanov, F.V. Hajieva, A.M. Maharramov, et al., J. Polym.-Plast. Technol. Eng. 57, 449 (2018).

[15] I.N. Bronshtein, K.A. Semendyayev, G. Musiol, H. Mühlig, Handbook of Mathematics, Springer, Berlin 2007, p. 1164.
[16] J. Heintzenberg, Aerosol Sci. Technol. 21, 46 (1994).

[17] Ya.I. Gerasimov, V.P. Dreving, E.N. Eremin, A.V. Kiselev, V.P. Lebedev, G.M. Panchenkov, A.I. Shlygin, in: Textbook of Physical Chemistry, Vol. 1, Ed. Ya.I. Gerasimova, Goskhimizdat, Moscow 1963, p. 543.

[18] M.A. Ramazanov, R.A. Alizade, A.M. Maharramov, F.V. Hajiyeva, J.R. Sultanova, H.A. Shirinova, J. Inorg. Organomet. Polym. Mater. 28, 2179 (2018).

[19] A. Gaul, D. Emmrich, T. Ueltzhöffer, Beilstein J. Nanotechnol. 9, 2968 (2018).

[20] G.A. Korn, T.M. Korn, Mathematical Handbook for Scientists and Engineers: Definitions, Theorems, and Formulas for Reference and Review (Dover Civil and Mechanical Engineering), Nauka, Moscow 1973, p. 832.

[21] T. Ishizaki, Nanomaterials (Basel) 6, 172 (2016).

[22] B. Issa, Int. J. Mol. Sci. 14, 21266 (2013).

[23] Guandong Zhang, Mater. Sci. Eng. C 30, 92 (2010).

[24] J.L. Wilson, J. Appl. Phys. 95, 1439 (2004).

[25] Hui Zhang, Dechang Zeng, Zhongwu Liu, J. Magn. Magn. Mater. 322, 2375 (2010).

[26] E. Chitra Devi, I. Soibam, J. Supercond. Novel Magn. 32, 1293 (2019).

[27] N.S. Bakhvalov, N.P. Zhidkov, G.M. Kobelkov, Numerical Methods, 6th ed., BKL Publ., 2008.

[28] V.I. Krylov, V.V. Bobkov, P.I. Monostyrny, Computational Methods, Vol. 2, Nauka, Moscow 1977 (in Russian).

[29] J. Mohapatra, Meiying Xing, J. Ping Liu, Materials 12, 3208 (2019).

[30] R. Gautam, D.B. Prabhu, V. Chandrasekaran, R. Gopalan, G. Sundararajan, J. Magn. Magn. Mater. 493, 165743 (2020).

[31] Guoping Zhao, F. Morvan, Xiulin Wan Rev. Nanosci. Nanotechnol. 3, 227 (2014). 